\title{
Is charm the key to understanding diffraction in DIS ?
}

\author{
M.F. McDermott \\ DESY, Notkestrasse 85, D-22603 Hamburg, Germany
}

\begin{abstract}
This talk concerns the production of open charm in diffractive deep inelastic scattering. This has been calculated recently in the context of the semi-classical approach to diffraction. A comparison is made to approaches in which the diffractive exchange is modelled by the exchange of two gluons in the $t$-channel. Two phenomenological test of the underlying partonic process are discussed.
\end{abstract}

The first results on the production of open charm in diffractive DIS were reported by the H1 collaboration [1], for the 1994 running period, last summer. Results from $\mathrm{H} 1$ and Zeus were given at this conference [2]. The experimental signature is a particular decay channel of $D^{*}$ mesons with a $p_{\perp}$ above $1 \mathrm{GeV}$; so far about 30 events have been found in total but this is expected to increase to at least 100 with the 1996 data. The heavy charm quark mass permits a reliable calculation within perturbative QCD. By examining exclusive channels such as open charm production it is hoped that more can be learned about the total diffractive sample in DIS.

The simplest QCD model one can think of for diffraction is the exchange of two gluons in a colour singlet in the $t$-channel. Several recent papers [3] present predictions for diffractive charm which have this mechanism of diffractive exchange in common. The leading order graph is shown in Fig.(1). The black blob in the figure represents the different ways in which higher order corrections and the gluon propagators have been considered in the various approaches. Unfortunately a complete $\mathcal{O}\left(\alpha_{s}\right)$ calculation, which would also include a gluon in the final state is not yet available. In terms of the diffractive structure function, these corrections are expected to be important when the diffractive mass is large compared to $Q^{2}$. More exclusive predictions for diffractive charm production are only available for the pure $c \bar{c}$ diffractive final state. The differential cross sections for transversely and longitudinally polarized photons can be written, in the double leading logarithmic approximation in terms of the square of the gluon density, $G(\xi)$, as follows 


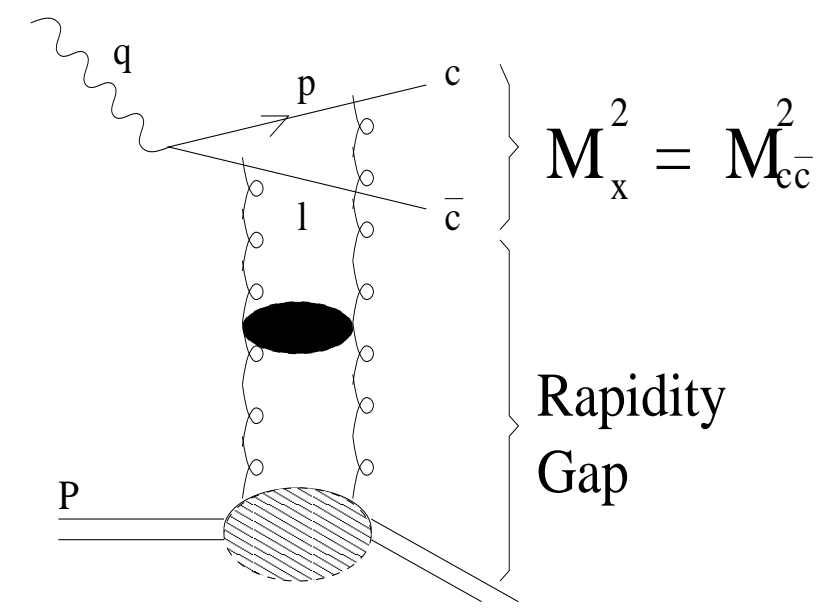

FIGURE 1. Two gluon exchange in the $t$-channel.

$$
\begin{aligned}
\frac{d^{2} \sigma_{L}}{d \alpha d p_{\perp}^{2}} & =\frac{2 e_{c}^{2} \alpha_{e m} \alpha_{s}^{2} \pi^{2}[\xi G(\xi)]^{2} C}{3\left(a^{2}+p_{\perp}^{2}\right)^{6}}[\alpha(1-\alpha)]^{2} Q^{2}\left(a^{2}-p_{\perp}^{2}\right)^{2} \\
\frac{d^{2} \sigma_{T}}{d \alpha d p_{\perp}^{2}} & =\frac{e_{c}^{2} \alpha_{e m} \alpha_{s}^{2} \pi^{2}[\xi G(\xi)]^{2} C}{6\left(a^{2}+p_{\perp}^{2}\right)^{6}}\left[4\left(\alpha^{2}+(1-\alpha)^{2}\right) p_{\perp}^{2} a^{4}+m_{c}^{2}\left(a^{2}-p_{\perp}^{2}\right)^{2}\right] \\
a^{2} & =\alpha(1-\alpha) Q^{2}+m_{c}^{2}
\end{aligned}
$$

where $\alpha$ is the momentum fraction of the virtual photon carried by the quark, $p_{\perp}^{2}$ is its transverse momentum squared and $\xi$ (or $x_{\mathbb{P}}$ ) is the longitudinal momentum fraction lost by the elastically scattered proton. The factor $C$ parameterizes the required extrapolation from $t \approx 0$ to the integrated cross section, $C \approx \Lambda_{Q C D}^{2}$.

An alternative view of diffraction, known as the semiclassical approach, which is very close in spirit to the aligned jet model has been developed recently [4]. In this proton rest frame calculation, leading twist diffraction results from the scattering of asymmetric partonic fluctuations of the virtual photon from the soft colour field of the proton (i.e. those configurations containing a parton which carries only a small fraction of the photon longitudinal momentum). The presence of this 'wee' or 'slow' parton, which necessarily has a low $p_{\perp}$, allows the fluctuation to develop a large transverse size by the time it arrives at the proton.

Configurations in which the wee parton is a charm quark are suppressed by powers of the charm mass, these include the contributions from the leading order $c \bar{c}$ configuration and the corresponding QCD Compton graphs. The dominant contribution is $\mathcal{O}\left(\alpha_{s}\right)$ and contains a wee gluon (cf. Fig.(2)). Expressed in terms of the momentum fraction, $\alpha^{\prime}$, and transverse momentum, $k_{\perp}^{\prime 2}$, of the gluon the differential cross sections are given by [5] 


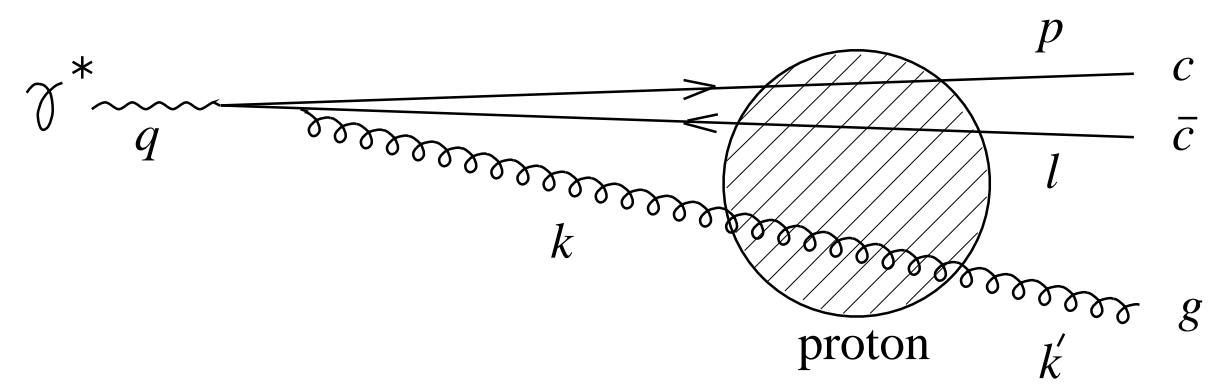

FIGURE 2. Dominant partonic process for diffractive charm production in the semiclassical approach.

$$
\begin{aligned}
\frac{d \sigma_{L}}{d \alpha d p_{\perp}^{2} d \alpha^{\prime} d k_{\perp}^{\prime 2}} & =\frac{e_{c}^{2} \alpha_{e m} \alpha_{s}}{16 \pi^{2}} \frac{\alpha^{\prime} Q^{2} p_{\perp}^{2}}{[\alpha(1-\alpha)]^{2} N^{4}} f^{\mathcal{A}}\left(\alpha^{\prime} N^{2}, k_{\perp}^{\prime}\right) \\
\frac{d \sigma_{T}}{d \alpha d p_{\perp}^{2} d \alpha^{\prime} d k_{\perp}^{\prime 2}} & =\frac{e_{c}^{2} \alpha_{e m} \alpha_{s}}{128 \pi^{2}} \frac{\alpha^{\prime}\left\{\left[\alpha^{2}+(1-\alpha)^{2}\right]\left[p_{\perp}^{4}+a^{4}\right]+2 p_{\perp}^{2} m_{c}^{2}\right\}}{[\alpha(1-\alpha)]^{4} N^{4}} f^{\mathcal{A}}\left(\alpha^{\prime} N^{2}, k_{\perp}^{\prime}\right) \\
f^{\mathcal{A}}\left(\alpha^{\prime} N^{2}, k_{\perp}^{\prime}\right) & =\int_{x_{\perp}}\left|\int \frac{d^{2} k_{\perp}}{(2 \pi)^{2}}\left(\delta^{i j}+\frac{2 k_{\perp}^{i} k_{\perp}^{j}}{\alpha^{\prime} N^{2}}\right) \frac{\operatorname{tr} \tilde{W}_{x_{\perp}}^{\mathcal{A}}\left(k_{\perp}^{\prime}-k_{\perp}\right)}{\alpha^{\prime} N^{2}+k_{\perp}^{2}}\right|^{2} \\
N^{2} & =Q^{2}+\frac{p_{\perp}^{2}+m_{c}^{2}}{\alpha(1-\alpha)}
\end{aligned}
$$

The heavy charm mass ensures that the $c \bar{c}$-pair stays small in transverse space and behaves like a gluon terms of colour. The superscript $\mathcal{A}$, which stands for adjoint, is written here since we are effectively testing the protons field with two "gluons". The eikonal factor $W^{\mathcal{A}}$ parameterizes the interaction of the system of partons with the proton and depends in general on the transverse momentum lost by the wee parton. Taking its trace projects onto the colour singlet configurations relevant for diffraction. Integration over the final state variables of the wee parton in the leading $\ln (1 / x)$ approximation gives

$$
\begin{aligned}
\frac{d \sigma_{L}}{d \alpha d p_{\perp}^{2}} & =\frac{e_{c}^{2} \alpha_{e m} \alpha_{s} \ln (1 / x) h_{\mathcal{A}}}{2 \pi^{3}\left(a^{2}+p_{\perp}^{2}\right)^{4}}[\alpha(1-\alpha)]^{2} Q^{2} p_{\perp}^{2} \\
\frac{d \sigma_{T}}{d \alpha d p_{\perp}^{2}} & =\frac{e_{c}^{2} \alpha_{e m} \alpha_{s} \ln (1 / x) h_{\mathcal{A}}}{16 \pi^{3}\left(a^{2}+p_{\perp}^{2}\right)^{4}}\left[\left(\alpha^{2}+(1-\alpha)^{2}\right)\left(p_{\perp}^{4}+a^{4}\right)+2 p_{\perp}^{2} m_{c}^{2}\right] \\
h_{\mathcal{A}} & =\int_{y_{\perp}} \int_{x_{\perp}} \frac{\left|\operatorname{tr} W_{x_{\perp}}^{\mathcal{A}}\left(y_{\perp}\right)\right|^{2}}{y_{\perp}^{4}}
\end{aligned}
$$

where $y_{\perp}$ is the transverse separation between the gluon and the $c \bar{c}$ pair.

If one boosts this system to the Breit frame, the initial state, off-shell, 'slow' gluon turns around and may be reinterpreted as an incoming gluon 


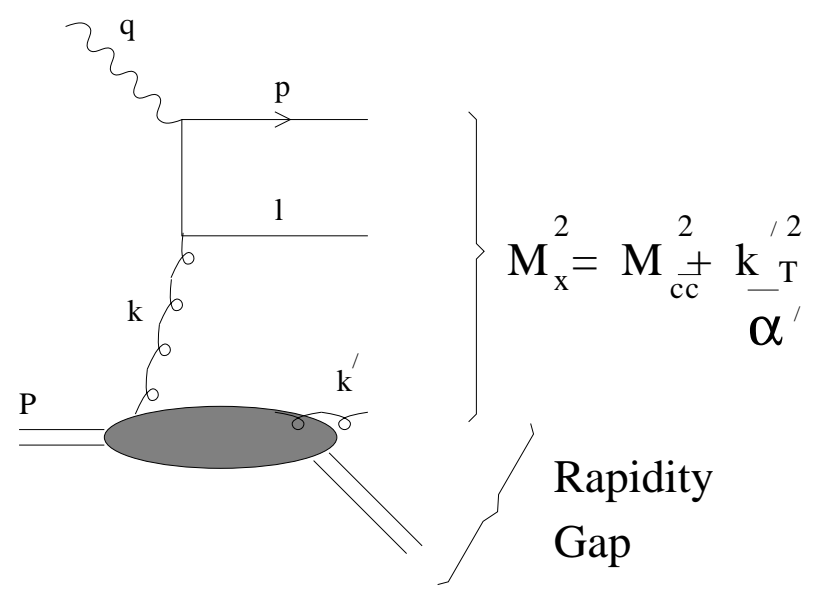

FIGURE 3. Boson-gluon fusion, with a final state gluon, in the Breit frame

of momentum fraction $y$ which lies between $\xi$ and $x[6]$. In this frame we have boson-gluon fusion off the diffractive gluon density in the proton [7] with an additional final state gluon (cf. Fig.(3)). The $p_{\perp}^{2}$ spectra is then logarithmically distributed between $m_{c}^{2}$ and $Q^{2}$. This should be contrasted to the much softer spectra expected from the two gluon exchange graphs where typically $p_{\perp}^{2} \sim m_{c}^{2}$. Note the above cross sections are also enhanced by a logarithm at small $x$ over configurations with a wee quark. In addition the constant, $h_{\mathcal{A}}$, is expected to be considerably bigger than its equivalent in the fundamental representation $\left(h_{\mathcal{A}} \approx 16 h_{\mathcal{F}}\right)$ providing a further enhancement [5].

In order to distinguish which type of graph is dominant we propose two phenomenological tests which reveal the nature of the underlying process and reflect the mechanism of colour neutralization which produces the different diffractive final states. Firstly, one may ask how many diffractive charm events survive above a given minimum $p_{\perp}^{2}$. To examine this we plot the quantity

$$
\sigma\left(p_{\perp, \min }^{2}\right)=\int_{p_{\perp, \min }^{2}}^{\infty} d p_{\perp}^{2} \int_{0}^{1} d \alpha \frac{d^{2} \sigma}{d p_{\perp}^{2} d \alpha}
$$

in Fig.(4).

We may also examine the expected mass spectra for the different final states. In Fig. (5) a normalized mass spectra comparing the mass of the charm pair, $M_{c \bar{c}}$, with the total diffractive mass, $M$, is shown. For the $c \bar{c}$ final state of Fig.(1) $M_{c \bar{c}}=M$, up to hadronization effects, and is represented by a block, where the width of the block represents a guess at the experimental uncertainty of diffractive mass measurement. The other curves in the figure represent the $c \bar{c} g$ final state of Figs. $(2,3)$ for which the mass of the pair may be considerably less than the total diffractive mass, as a result of the 'slow' gluon in the final state. Details abut how the latter curves were arrived at and the meaning of $C_{s}$ are given in [5]. 


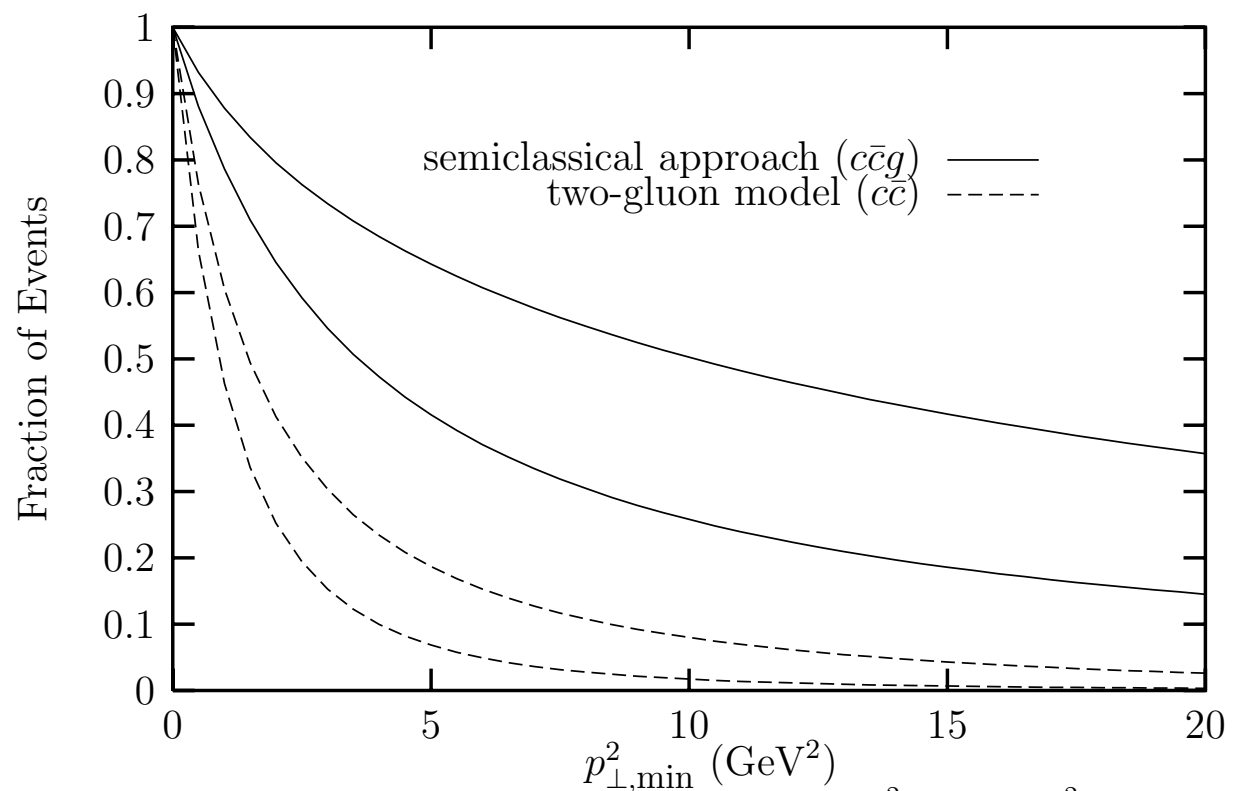

FIGURE 4. The fraction of diffractive charm events above $p_{\perp, \min }^{2}$ for $Q^{2}$ of $10 \mathrm{GeV}^{2}$ and $100 \mathrm{GeV}^{2}$ (lower and upper curve in each pair).

An additional means of distinguishing the underlying diffractive mechanism is the energy dependence of the two processes. In the two gluon model a steeply rising gluon density, taken from a fit to $F_{2}$ for example, produces a rise in diffractive charm events that is twice as steep with energy. In contrast the semiclassical approach the energy dependence is flat, at least at this order, corresponding to a classical bremstrahl spectrum of gluons.

Given the increase in statistics of Hera for the 1996 running period, it is hoped that these phenomenological tests may be performed very soon.

\section{REFERENCES}

1. H1 collab., pa02-060, A Measurement of the Production of $D^{* \pm}$ Mesons in DeepInelastic Diffractive Interactions at HERA, XXVIII ICHEP, Warsaw, 1996

2. C. Cormack, H1 collab., these proceedings; J Terron, ZEUS collab., these proceedings.

3. E.M. Levin, A.D. Martin, M.G. Ryskin, and T. Teubner, hep-ph/9606443; M. Genovese, N.N. Nikolaev, B.G. Zakharov, Phys. Lett. B378 (1996) 347; H. Lotter, preprint DESY 96-260, hep-ph/9612415; M. Diehl, preprint CPTH-S4920197, hep-ph/9701252

4. W. Buchmüller and A. Hebecker, Nucl. Phys. B476 (1996) 203; W. Buchmüller, M.F. McDermott, and A. Hebecker, Nucl. Phys. B487 (1997) 283, erratum ibid.

5. W. Buchmüller, M.F. McDermott, and A. Hebecker, hep-ph/9703314.

6. A. Hebecker, preprint DAMTP-97-10, hep-ph/9702373. 


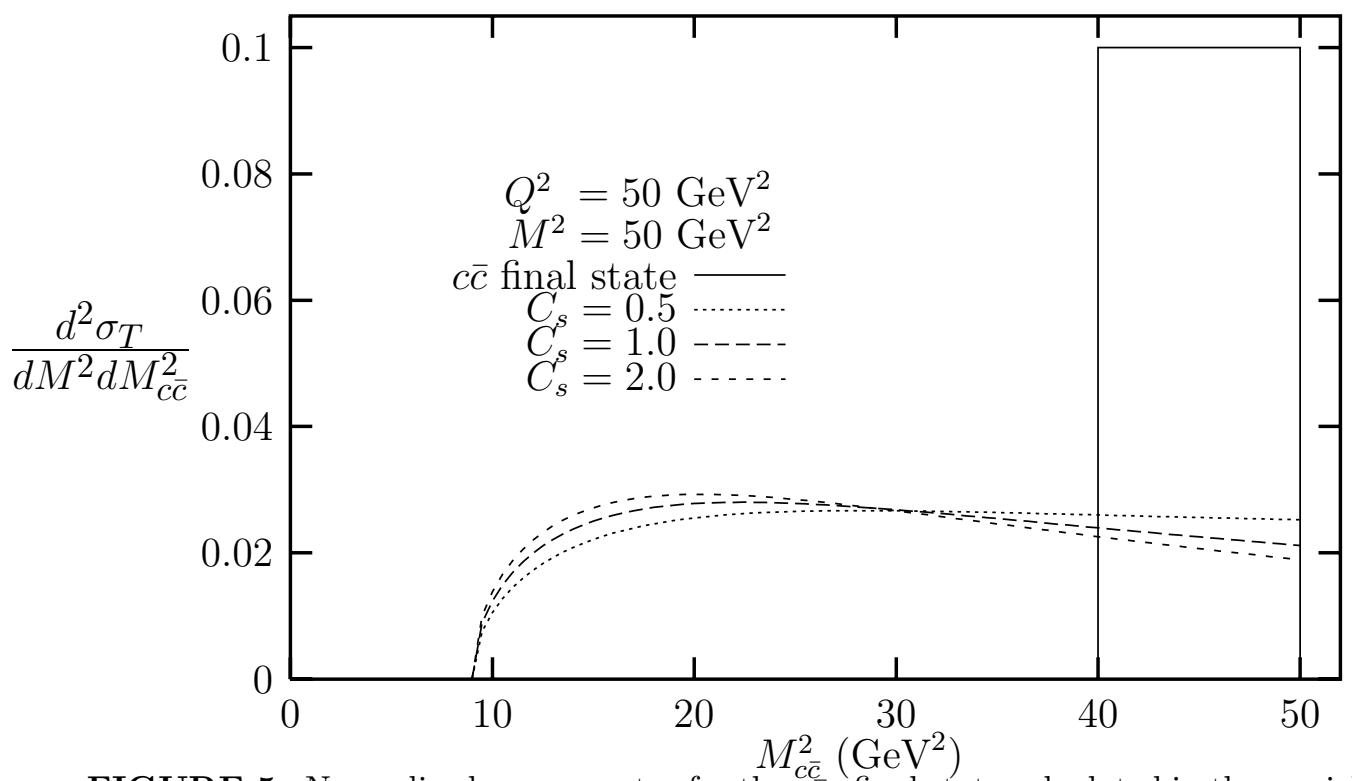

FIGURE 5. Normalized mass spectra for the $c \bar{c} g$ final state calculated in the semiclassical approach and the $c \bar{c}$ final state calculated in the two gluon exchange models.

7. A. Berera and D. E. Soper, Phys Rev D53 (1996) 203; Phys Rev D50 (1994) 4328. 